\title{
Systematic literature survey: applications of LoRa communication
}

\author{
Lone Kolobe, Boyce Sigweni, Caspar K Lebekwe \\ Department of Electrical, Computer and Telecommunications Engineering, \\ Botswana International University of Science and Technology, Botswana
}

\begin{tabular}{l} 
Article Info \\
\hline Article history: \\
Received Mar 2, 2019 \\
Revised Dec 5, 2019 \\
Accepted Dec 10, 2019
\end{tabular}

Keywords:

LoRa

LoRa survey

LoRa applications botswana

LoRaWAN

LPWAN

\begin{abstract}
LoRa is a communication scheme that is part of the low power wide area network (LPWAN) technology using ISM bands. It has seen extensive documentation and use in research and industry due to its long coverage ranges of up-to $20 \mathrm{~km}$ or more with less than $14 \mathrm{~dB}$ transmit power. Moreover, some applications report theoretical battery lives of up to 10 years for field deployed modules utilising the scheme in wireless sensor network (WSN) applications. Additionally, the scheme is very resilient to losses from noise, as well as bursts of interference through its forward error correction (FEC) scheme . Our objective is to systematically review the empirical evidence of the use-cases of LoRa in rural landscapes, metrics and the relevant validation schemes. In addition, the research is evaluated based on (i) mathematical function of the scheme (bandwidth use, spreading factor, symbol rate, chip rate and nominal bit rate) (ii) usecases (iii) test-beds, metrics of evaluation and (iv) validation methods. A systematic literature review of published refereed primary studies on LoRa applications was conducted using articles from 2010-2019. We identified 21 relevant primary studies. These reported a range of different assessments of LoRa with 10 out of 21 reporting on novel use cases. The authors conclude that more work is needed in terms of field testing, as no articles could be found on performance/deployment in Botswana or South Africa despite the existence of LoRa networks in both countries. Thus researchers in the region can research propagation models performance, the energy efficiency of the scheme and MAC layer as well as the channel access challenges for the region.
\end{abstract}

Copyright (C) 2020 Insitute of Advanced Engineeering and Science. All rights reserved.

\section{Corresponding Author:}

Lone Kolobe,

Department of Electrical, Computer and Telecommunications Engineering,

Botswana International University of Science and Technology,

Private Bag 16, Khurumela, Palapye, Botswana.

Email: lone.kolobe@studentmail.biust.ac.bw

\section{INTRODUCTION}

By the year 2020, it is estimated that there will be over 50 billion devices online [1]. On a similar note, at least 25 billion devices communicating on a machine to machine (M2M) level are projected [2] to be part of the internet of things (IoT). IoT technology is deployed in the network layer [3]. Communication technologies which have dominated in the long range IoT communication landscape are: Sigfox, LoRa and NB-IoT [1, 2, 4, 5]. The two former schemes, Sigfox and LoRa, both transmit over the sub $1 \mathrm{GHz}$ ISM bands while the latter (NB-IoT) utilizes LTE and 3GPP technology thus transmitting in the licensed band [6]. This article gives a systematic review of the empirical evidence of the use-cases of LoRa in rural landscapes, metrics and the relevant validation schemes. In addition, the research is evaluated based on (i) mathematical function of the scheme (bandwidth use, spreading factor, symbol rate, chip rate and nominal bit rate) (ii) use-cases (iii) test-beds, metrics of evaluation and (iv) validation methods. 


\subsection{LoRa and LoRaWAN summary}

LoRa is a proprietary spread spectrum modulation scheme that is derivative of Chirp Spread Spectrum modulation (CSS) and which trades data rate for sensitivity within a fixed channel bandwidth [7]. This technology also transmits in the sub 1GHz ISM bands [8]. The underlying technology (protocols) was developed by the LoRa alliance, a group of the most influential companies in the global telecommunications industry [2] including but not limited to IBM and SEMTEC $[9,10]$ to name a few. LoRa utilizes proprietary chirp spread spectrum (CSS) modulation [11]. A result of this modulation scheme gives LoRa higher resilience to interference, as well as noise and makes it difficult to join or even detect $[8,1]$. Additionally, LoRa utilizes six (6) spreading factors (SF7 to SF12), to allow the scheme to adapt the data rate and range [1]. This also allows the sent data packet to be padded making them more resilient to noise. However, latency is gained in employing higher spreading factors $[11,10]$. It is worth noting that the LoRa technology like many others in the LPWAN class utilizes a "wait and listen" scheme [6,12] making it half duplex. Using the LoRaWAN protocol, LoRa has a message payload of up to 243bytes [1]. As a means of addressing numerous potential use cases in IoT communication, LoRa has three classes of communication which all keep the core characteristics of LPWANS while adding flexibility for cases where constant communication is needed these are classes A, B and C $[1,6]$.

LoRa also has a MAC protocol (LoRaWAN) developed by SEMTEC and IBM that specifies the connection properties of the LoRa modulation scheme while also allowing the automatic or manual setting of connection parameters [11]. More to this, LoRaWAN uses a scheme -adaptive data rate (ADR)- to set the connection parameters. LoRa PHY layer uses a series of up and down chirp signals to modulate and transmit signals over the air [11]. Spread spectrum techniques have enjoyed significant attention due in no small part to their resistance to jamming [13]. A technique is considered spread spectrum if the transmission bandwidth is larger than the minimum required information transmission bandwidth [13, 14]. Furthermore, the following criteria were outlined by [13] as the properties of spread spectrum modulation:

(a) The bandwidth used for transmission is much greater than the minimum bandwidth required to transmit information [13].

(b) The "spreading" process is done by a "spreading signal or code signal". This signal is independent of the data being transmitted.

(c) At the receiver, signal recovery is performed through correlation of the spread signal with a replica of the coding signal used to spread the transmitted data [13].

The benefits of increasing the bandwidth of the channel are overall improved performance. However, this is not guaranteed as the bandwidth is increased to infinity. Refer to 1.

$$
\begin{aligned}
& C=B W * \log _{2}\left(1+\frac{S}{N}\right) \\
& T_{s}=\frac{2^{S F}}{B W}, \text { seconds } \\
& R_{s}=\frac{1}{T_{\mathrm{s}}}=\frac{B W}{2^{S F}}, \text { Symbols per second } \\
& R_{c}=R_{s} * 2^{S F}, \text { Chips per second } \\
& R_{b}=S F * \frac{\frac{4}{4+C R}}{\frac{2^{S F}}{B W}}, \text { Bits per second }
\end{aligned}
$$

Where:

(a) $\mathrm{SF}=$ spreading factor, ranging from 7-12. Spreading factors denote the number of chirps used to encode data bits [15]. Where the number of chirps will be $=2^{S F}$ [15].

(b) $\mathrm{CR}=$ coding rate, ranging from 1-4. This specifically used by LoRa fro forward error correction used to protect tramissions from error bursts [11]. Coding rate can be set to $4 / 5,4 / 6,4 / 7$ or $4 / 8$, in doing this higher CR will give better protection while increasing the packet time on air [11].

(c) $\mathrm{BW}=$ modulation bandwidth in hertz, $T_{s}=$ symbol period, $R_{s}=$ symbol rate, $R_{c}=$ chip rate, $R_{b}=$ nominal bit rate [7]. 


\section{RESEARCH METHOD}

The authors used the search protocol in Table 1 from [16] to find the literature pertaining to the subject matter. Data utilised in the research was obtained from articles in line with search requirements. This allowed for the assessment of popular models on grander scales IE across continents.

\subsection{Research questions}

Through a combination of literature review, observation of hobbyist chatrooms, OEM white papers and conference material, a number of research questions are poised to be answered.

Table 1. Systematic literature review summary

\begin{tabular}{ll}
\hline Characteristic & Value \\
\hline Review type & Systematic literature review \\
Research question(s) & $\begin{array}{l}\text { What is Lora and how does the PHY layer function(RQ1), What is the diversity and range of } \\
\text { the LoRa scheme use cases?(RQ2) How is LoRa network performance measured ? } \\
\text { Guide researchers and practitioners using/intending to use LoRa on the limitations and ideal } \\
\text { use cases. }\end{array}$ \\
Purpose & Automated and hand search, citation analysis, previously known articles, \\
& IJECE, IEEE Xplore, ScienceDirect, Google scholar, ACM digital library, Springer \\
Search method & Empirical studies relating to LoRa and LoRaWAN applications, tests and use cases \\
Databases used & Experiments, case studies, observational studies, simulation. \\
Population & (i) Refereed paper (journal or conference) (ii) Empirical study (iii) Copy of the article available \\
Study types & in the English language \\
Inclusion criteria & $07 / 01 / 19-$ present \\
& $2010-2019$ plus in press articles \\
Search date &
\end{tabular}

\subsubsection{RQ1: What is the diversity and range of the LoRa scheme use cases?}

Rationale: research question 2 will establish the different applicable use cases of LoRa technology. Furthermore it will establish the criterion used to determine the appropriateness of a given LPWAN technology in a specific area.

\subsubsection{RQ2: How is LoRa network performance measured?}

Rationale: Performance measurement in this context will specify the means by which deployment is tested (test-beds). Moreover, this research question will allude to the metrics used and the validation methods as ranked by prevalence.

\subsection{Search strategy}

Previous articles on LPWAN technology have mainly focused on documented use case scenarios whether academic or commercial. Additionally, for most articles used in this study, care was taken to derive performance reports from OEM white-papers as well as journals, and conference articles/papers pertaining to the investigation LPWAN practical performance limitations. Lastly, novel use cases and performance experiences were solicited from Medium, which is an online repository of DIY and hobbyist practical exploits of LPWAN SDR and MAC layer manipulation.

\subsection{Search terminology}

The following search terminology was used to ascertain journal, article and white-paper information on the subject areas. We employed the following steps to build the search terms based on existing literature and material we found insufficiently in our research.

(a) Derive major terms from the research questions.

(b) Identify synonyms and alternative spellings for major terms.

(c) Using the Boolean OR and AND to incorporate and link alternative spellings and synonyms.

The search terminology development steps above resulted in the establishment of the following search strings.

“(LoRa OR LPWAN OR LoRaWAN Propagation Model(s) OR Performance OR Path loss) AND/OR (433MHz/868MHz band LPWAN OR Low power Wide Area OR Low Power Area). (SNR OR Path loss Performance OR BER OR Propagation OR PRR OR RSSI). (Narrowband OR Chirp Spread Spectrum AND/OR LPWAN OR LoRa OR Long Range) AND(Path loss OR Propagation)" 


\subsection{Search process}

The following process was used to search, filter and store material from various journals. A three (3) step process is outlined below:

(a) Step 1 : Enter search terms in google scholar/IEEE xplore/Springer/ACM/Elsivier/MDPI/Wiley Online sources/IJECE. Apply forward search via citations in search results, keep material with relevant timeline 2010-2018. Search titles of found journals for relevance. Download filtered journal with satisfactory keywords and title

(b) Step 2 : Review abstract of Step 1 filtered material. Keep document that is relevant according to contents of abstract. Use references in accepted papers to determine/find new material.

(c) Step 3 : Identify new articles relevance in accordance with Step 2, note duplicates and return to Step 1.

\section{RESULT}

\subsection{RQ1: What is the diversity and range of the LoRa scheme use cases?}

LoRa boasts resilience to noise, multipath fading, doppler shift resistance as well as long range transmission rates [7]. J. M. Marais, et al. in [17] observed numerous indoor and outdoor applications. For context the largest global open source network of LoRa gateways is currently "TheThingsNetwork" [15]. "TheThingsNetwork" can also be regarded as the application layer allowing for management of data collected from the perception layer and routed through the network layer as described by [3]. Some of the most novel use cases observed in literature are provided in Table 2.

Table 2. LoRa and LoRaWAN use cases

\begin{tabular}{|c|c|c|}
\hline Source & Application Summary & Class \\
\hline$[15]$ & $\begin{array}{l}\text { UK flood network. A series of water level sensors deployed } \\
\text { across rivers around the UK to alert residents of dangerous wa- } \\
\text { ter levels and flood risks. }\end{array}$ & Real time monitoring \\
\hline$[18]$ & $\begin{array}{l}\text { The authors documented their findings in the use of LoRa for } \\
\text { tractical tracking systems in Thailand. }\end{array}$ & Real time covert monitoring \\
\hline [19] & $\begin{array}{l}\text { The researchers were able to find positive results with a man- } \\
\text { grove monitoring. This was done through the transmission of } \\
\text { images over the LoRa physical layer with a custom encryption } \\
\text { technique. }\end{array}$ & Multimedia and agriculture monitoring \\
\hline [20] & $\begin{array}{l}\text { Carlsson et al investigated deployment of LoRa at the } 868 \mathrm{MHz} \\
\text { frequency in three different scenarios: dense forest, open set- } \\
\text { ting and high elevation. The experiment illustrates the LoRa } \\
\text { physical layer and LoRaWAN resilience in various settings. }\end{array}$ & Performance evaluation \\
\hline [21] & $\begin{array}{l}\text { Juha et al were able to perform experiments on the effects of } \\
\text { moving receivers on both land and water in urban and indus- } \\
\text { trial environments. These settings are the city of Oulu in Fin- } \\
\text { land as well as a dock in the same area. }\end{array}$ & Industrial monitoring \\
\hline
\end{tabular}

F. Adelantado, et al. [22] discusses that LoRa technology is limited in applications, mainly those with a tolerance to any jitters. This eliminates applications needing constant feed of multimedia. Additionally, the authors correctly point out the limitations of LoRa in so far as real-time metering is concerned as the duty cycle limitations imposed on the ISM band usage. However, due to the varied number of classes and the reliability added when there are more gateways, refer to section 1.1, the latter is debatable. It is also worth noting that areas of industrial control will remain out of reach as with the lowest SF, equation 2 shows that the delay will be $40 \mathrm{~ms}$ before any control signal is received.

Nonetheless, the fact that LoRa's MAC layer can be easily modified opens the door to multiple use cases. This is observed when authors of [23] created LoRaBink for energy efficient multi-hop transmission. Similarly, with smart encoding techniques transmission of images is possible.

\subsection{RQ2: How is LoRa network performance measured?}

This research question will draw attention to how the performance of various deployments observed in literature was done with emphasis on validation, test-bed and metrics. Jaco M. Marais et al [24] authored a 
paper summerising the various testbeds used to test LoRa and LoRaWAN performance. Network performance for LoRa is conducted on the MAC layer's ability to transfer packets reliably. Performance is measured on the following: Packet reception rate (PRR) and packet error rate (PER), the data extraction rate (DER) and percentage loss of packets [25]. Additionally, the physical layer's performance is often gauged based on the received signal strength indicator (RSSI), signal to noise ratio (SNR) and the bit error rate (BER) as well as the network energy consumption (NEC) [25].

\subsubsection{Intra-scheme metrics of comparison}

The metrics on which LoRa and the next widely available schemes (Sigfox) are to be compared are the availability of QoS, node battery life, packet latency, scalability, payload length, network coverage, global adoption, cost of deployment and the ability to be open source.

(a) QoS : Sigfox and LoRa have very poor QoS. However, LoRa through the LoRaWAN protocol offsets this by broadcasting information to all nearby base stations in essence increasing the degrees of diversity [1]. Sigfox will similarly transmit its message multiple times with no acknowledgements (typically three times by default) [1].

(b) Battery life and latency : Sigfox and LoRa have end nodes consuming lower energy through the use of function states. The exception in this case being LoRa class $\mathrm{C}$ devices. Similarly, LoRa class $\mathrm{C}$ having better latency compared to class A, B as well as Sigfox [1].

(c) Scalability and payload length : Sigfox and LoRa base stations can handle 50 thousand devices each per base station [1] making them fairly evenly matched in this regard. More to this, LoRa and Sigfox have 243 bytes [10] and 12 bytes [1] respectively with the latter characteristic tipping the favor to LoRa.

(d) Network coverage and range : Sigfox commands the longest range, with line of sight (LoS) communication capable of effective transmission at $40 \mathrm{~km}$ [1]. The second longest range with LoS range of approximately $20 \mathrm{~km}$ is LoRa [8].

(e) Global adoption : According to [1] LoRa is deployed across 42 countries while Sigfox is only available in 31. Additionally, LoRa allows communication hybridization by using different communication schemes to transmit data effectively over a given distance.

(f) Cost: The hardware cost associated with Sigfox is significantly high due to the none liberalized hardware ecosystem [1]. However, the LoRa Alliance has seen hardware development of LoRa from other organizations such as Ada-fruit and STM significantly improving availability of both enabled devices and public gateways $[1,2]$.

(g) Open source: Considerable work has gone on to allowing this on the LoRa modulation scheme. The Sigfox ecosystem remains under the tight control of the creators of Sigfox. Whereas SEMTEC's LoRa continues to enjoy widespread support and experimentation of hobbyists and professionals alike $[26,6,1]$.

\subsubsection{LoRa performance metrics and test-beds}

The performance metrics of LoRa and LoRaWAN along with the validation method are shown in Table 3 .

Table 3. LoRa and LoRaWAN performance metrics, validation

\begin{tabular}{|c|c|c|c|}
\hline Source & Test-bed & Performance metrics & Validation method \\
\hline [27] & $\begin{array}{l}\text { Semtech SX1307 transceiver as a gateway, Lo- } \\
\text { RaWAN testing done using Multitech Mdot de- } \\
\text { vices in the } 868 \mathrm{MHz} \text { band }\end{array}$ & RSSI & Project monitoring \\
\hline [28] & TI C110 radio module in the $433 \mathrm{MHz}$ band & RSSI & Dynamic analysis, simulation \\
\hline [21] & $\begin{array}{l}\text { Kerlink LoRa IOT station D100-1000 antenna } \\
\text { (base station), and LoRa mote Semtech SX1272 } \\
\text { (mobile node) }\end{array}$ & $\begin{array}{l}\text { RSSI, Path-loss exponent, } \\
\text { path-loss intercept }\end{array}$ & Project monitoring \\
\hline [29] & $\begin{array}{l}\text { SX1272 LoRa sheild with arduino nucleo and } \\
\text { raspberry pi, IC } 880 \text { a concentrator and STMicro- } \\
\text { electronics Nucleo-L073RZ MCU board }\end{array}$ & RSSI, SNR, PRR & Project monitoring \\
\hline [30] & $\begin{array}{l}\text { RN248 (RX side), RHF25008 with SX1301 chip } \\
\text { (TX side) with a 5dBi antenna, OBCI smart ev- } \\
\text { erything fox board at the receiver }\end{array}$ & RSSI, PDR & Simulation, project monitoring \\
\hline [11] & Netblocks XRange SX1272 LoRa RF module & $\mathrm{PRR}, \delta_{i}, \mathrm{RSSI}$ & $\begin{array}{l}\text { Project monitoring and simula- } \\
\text { tion }\end{array}$ \\
\hline
\end{tabular}




\section{DISCUSSION}

Through this systematic literature review (SLR), the authors were able to find 13 articles to ground the study and answer the 3 research questions outlined in section 3 . The main findings of this study are the following:

(a) LoRa technology and LPWANs have a wide array of applications with an almost equal number of potential research areas mostly into spectral access, quality of service, and MAC layer modifications.

(b) The main reasons for which LoRa has bloomed in IOT and research spaces is invariably true as the scheme is able to achieve very long communication distances however there is a catch. Distilling performance into two areas the MAC layer and the PHY layer. Due to the MAC layers' limitations in terms of QOS and the static nature of the protocol significant performance maybe lost as a lot of testing must be done to establish which CR, BW and SF are best suited for a particular area to maximise PRR, NEC as well as DER. With regard to the physical layer, in all instances where little or no disruption of the Frensel zone occurs, signal propagation is observed to be high.The authors observed that it is possible to obtain relatively high levels of RSSI and still have significant PER invariably leading to inefficiency in the network implementation mainly due to poor settings at the MAC layer.

(c) Being an ISM based scheme, the duty cycle limitations of LoRa imposed by statutes poses significant bottlenecks in terms of accessibility as the ALOHA protocol means significant wait periods can be expected between transmissions in very RF active zones: urban and rural landscapes. This turns out to be a non-hindrance in the application area Botswana aspires for, namely agricultural monitoring, as most areas of interest have little RF traffic in the sub GHz ISM bands. However spectral measurements may need to be taken to confirm with a quantitative certainty in future.

(d) LoRa technology range tests/evaluations are predominantly used in the developed world. Thus the real world behaviour of LoRa is not documented in the Southern Africa although the number of gateways in the area is increasing. It is therefore the position of the authors that more field testing be done to adapt the ADR properties of the technology in Botswana and Southern Africa as a whole to ensure more efficient network deployments in agriculture and IOT novel applications.

(e) More work is needed to isolate appropriate pathloss and propagation models for any area. Several authors have observed the disconnect between real-world readings and traditional propagation models. This is especially true for those based on the Frensel equation and traditional path loss model. However, in all experiments it was noted that the height above ground is critical in improving range.

\section{CONCLUSION}

As with any SLR, the authors concede that the study may have not acquired all the relevant literature. Additionally, due to the number of papers/articles surveyed it is not possible to perform a meta analysis. Despite this limitation, we believe that the material reviewed has shone a considerable light to the area of LoRa and LPWANs while indicating a lack of research in LoRa in the region.

\section{REFERENCES}

[1] K. Mekki, E. Bajic, F. Chaxel, and F. Meyer, "A comparative study of LPWAN technologies for large-scale IoT deployment," ICT Express, 2018. [Online]. Available: https://doi.org/10.1016/j.icte.2017.12.005

[2] R. S. Sinha, Y. Wei, and S. H. Hwang, "A survey on LPWA technology: LoRa and NB-IoT," ICT Express, vol. 3, no. 1, pp. 14-21, 2017. [Online]. Available: http://dx.doi.org/10.1016/j.icte.2017.03.004

[3] A. Rghioui and A. Oumnad, "Internet of things: Surveys for measuring human activities from everywhere," International Journal of Electrical and Computer Engineering, vol. 7, no. 5, pp. 2474-2482, 2017.

[4] H. Ouldzira, A. Mouhsen, H. Lagraini, A. Tabyaoui, and M. Chhiba, "Smart monitoring information system based on RF 433 Mhz ( SMIS )," vol. 9, no. 6, pp. 5143-5149, 2019.

[5] G. Samara and M. Aljaidi, "Efficient energy, cost reduction, and QoS based routing protocol for wireless sensor networks," International Journal of Electrical and Computer Engineering, vol. 9, no. 1, pp. 496504, 2019. 
[6] R. HICKEY, "IOT / M2M PLATFORMS LPWAN : Choice Overload and Confusion," pp. 2-5, 2018. [Online]. Available: https://www.lightreading.com/iot/m2m-platforms/lpwan-choice-overloadand-confusion/a/d-id/718233

[7] Semtech, "LoRa ${ }^{\mathrm{TM}}$ Modulation Basics Semtech," Tech. Rep. May, 2015.

[8] T. Lassen, "Long-range RF communication: Why narrowband is the de facto standard," WHITE Pap. Texas Instruments, p. 8, 2014.

[9] N. Blenn and F. Kuipers, "LoRaWAN in the Wild: Measurements from The Things Network," 2017. [Online]. Available: http://arxiv.org/abs/1706.03086

[10] LoRaAlliance and LoRa Alliance, "LoRaWAN 1.1 Specification," LoRa Alliance, no. 1.1, p. 101, 2017. [Online]. Available: https://lora-alliance.org/resource-hub/lorawantm-specification-v11

[11] M. Bor and U. Roedig, "LoRa transmission parameter selection," Proceedings - 2017 13th International Conference on Distributed Computing in Sensor Systems, DCOSS 2017, vol. 2018-Janua, pp. 27-34, 2018.

[12] O. Kennedy, E. Chukwu, O. Shobayo, E. Noma-Osaghae, I. Okokpujie, and M. Odusami, "Comparative analysis of the performance of various active queue management techniques to varying wireless network conditions," International Journal of Electrical and Computer Engineering (IJECE), vol. 9, no. 1, p. 359, 2019.

[13] B. Sklar, Digital Communications Fundamentals and Applications 2nd Edition, 2nd ed. Prentice Hall, 2001, no. 1132.

[14] I. A. Hieder, "Compared to wireless deployment in areas with different environments," International Journal of Electrical and Computer Engineering, vol. 9, no. 2, pp. 934-940, 2019.

[15] N. Blenn and F. Kuipers, "LoRaWAN in the Wild: Measurements from The Things Network," 2017. [Online]. Available: http://arxiv.org/abs/1706.03086

[16] B. Sigweni and M. Shepperd, "Feature weighting techniques for CBR in software effort estimation studies: a review and empirical evaluation," in Proceedings of the 10th International Conference on Predictive Models in Software Engineering. ACM, 2014, pp. 32-41.

[17] J. M. Marais, R. Malekian, and A. M. Abu-Mahfouz, "LoRa and LoRaWAN testbeds: A review," 2017 IEEE AFRICON: Science, Technology and Innovation for Africa, AFRICON 2017, pp. 1496-1501, 2017.

[18] W. San-Um, P. Lekbunyasin, M. Kodyoo, W. Wongsuwan, J. Makfak, and J. Kerdsri, "A long-range low-power wireless sensor network based on U-LoRa technology for tactical troops tracking systems," in Proceedings - ACDT 2017: 3rd Asian Conference on Defence Technology: Advance Research Collaboration on Defence Technology, 2017, pp. 32-35.

[19] A. I. Akram H. Jebril , Aduwati Sali and M. F. A. Rasid, "Overcoming Limitations of LoRa Physical Layer in image transmission," MDPI, Sensors, vol. 18, no. 3257, pp. 1-22, 2018.

[20] O. Galinina, S. Andreev, S. Balandin, and Y. Koucheryavy, "Measuring a Lora Network: Performance, Possibilities and Limitations," Springer Nature (Switzerland), no. September, pp. 116-128, 2018. [Online]. Available: http://dx.doi.org/10.1007/978-3-030-01168-0_11

[21] J. Petäjäjärvi, K. Mikhaylov, A. Roivainen, T. Hänninen, and M. Pettissalo, "On the coverage of LPWANs: Range evaluation and channel attenuation model for LoRa technology," in 2015 14th International Conference on ITS Telecommunications, ITST 2015, 2016, pp. 55-59.

[22] F. Adelantado, X. Vilajosana, P. Tuset-Peiro, B. Martinez, J. Melia-Segui, and T. Watteyne, "Understanding the Limits of LoRaWAN," IEEE Communications Magazine, vol. 55, no. 9, pp. 34-40, 2017.

[23] M. C. Bor, J. Vidler, and U. Roedig, "LoRa for the Internet of Things," Proceedings of the 2016 International Conference on Embedded Wireless Systems and Networks, pp. 361-366, 2016. [Online]. Available: http://eprints.lancs.ac.uk/77615/

[24] J. M. Marais, R. Malekian, and A. M. Abu-Mahfouz, "LoRa and LoRaWAN testbeds: A review," 2017 IEEE AFRICON: Science, Technology and Innovation for Africa, AFRICON 2017, pp. 1496-1501, 2017.

[25] M. H. Habaebi, I. J. Chowdhury, M. R. Islam, and N. A. B. Zainal, "Effects of shadowing on LoRa LPWAN radio links," International Journal of Electrical and Computer Engineering, vol. 7, no. 6, pp. 2970-2976, 2017.

[26] P. P. Ray, “A survey on Internet of Things architectures,” J. King Saud Univ. - Comput. Inf. Sci., vol. 30, no. 3, pp. 291-319, 2018. [Online]. Available: https://doi.org/10.1016/j.jksuci.2016.10.003

[27] A. J. Wixted, P. Kinnaird, H. Larijani, A. Tait, A. Ahmadinia, and N. Strachan, "Evaluation of LoRa and LoRaWAN for wireless sensor networks,” in Proceedings of IEEE Sensors, vol. 0, 2017, pp. 5-7. 
[28] Á. Milánkovich, K. Lendvai, S. Imre, and S. Szabó, "Radio Propagation Modeling on 433 MHz," Springer, pp. 384-395, 2017.

[29] A. Carlsson, I. Kuzminykh, R. Franksson, and A. Liljegren, "Measuring a LoRa Network: Performance, Possibilities and Limitations," in Springer Nature (Switzerland). Springer International Publishing, 2018, no. September, pp. E1-E1. [Online]. Available: http://dx.doi.org/10.1007/978-3-030-01168-0_11 http://link.springer.com/10.1007/978-3-030-01168-0_65

[30] R. Sanchez-Iborra, J. Sanchez-Gomez, J. Ballesta-Viñas, M. D. Cano, and A. F. Skarmeta, "Performance evaluation of lora considering scenario conditions," Sensors (Switzerland), vol. 18, no. 3, 2018. 